\title{
Urinary bladder reconstruction using autologous collagenous connective tissue membrane "Biosheet" induced by in-body tissue architecture
}

Yasumasa limori

Osaka Prefecture University Graduate School of Life and Environmental Sciences https://orcid.org/0000-0003-2575-2287

Kazuma Naito

Osaka Prefecture University Graduate School of Life and Environmental Sciences

Ryosuke Iwai

Okayama University of Science Institute of Frontier Science and Technology

\section{Kengo Nagatani}

Osaka Prefecture University Graduate School of Life and Environmental Sciences

\section{Yuka Inoue}

Osaka Prefecture University Graduate School of Life and Environmental Sciences

\section{Marina Funayama-Iwai}

Osaka Prefecture University Graduate School of Life and Environmental Sciences

\section{Mari Okamoto}

Osaka Prefecture University Graduate School of Life and Environmental Sciences

Mio Nakata

Osaka Prefecture University Graduate School of Life and Environmental Sciences

Keiichiro Mie

Osaka Prefecture University Graduate School of Life and Environmental Sciences

Hidetaka Nishida

Osaka Prefecture University Graduate School of Life and Environmental Sciences

\section{Yasuhide Nakayama}

Biotube Co Ltd

Hideo Akiyoshi ( $\sim$ akiyoshi@vet.osakafu-u.ac.jp )

Research article

Keywords: In body tissue architecture, Biosheets, Biomaterial, Reconstruction, Bladder, Animal model

Posted Date: August 26th, 2019

DOI: https://doi.org/10.21203/rs.2.13584/v1 
License: (c) (i) This work is licensed under a Creative Commons Attribution 4.0 International License. Read Full License 


\section{Abstract}

Background: Tissue engineering technology has the potential for bladder reconstruction without complications. We have previously developed autologous collagenous prosthetic tissues using in-body tissue architecture (iBTA). This is a cell-free tissue engineering technology that can produce autologous implantable tissues be a desired shape by simple subcutaneous embedding of a specially designed mold. Grafts formed by iBTA can be made in any shape and form, including sheet-shaped tissues (Biosheet). In this study, we evaluated the efficacy and safety of autologous Biosheet as bladder repair material in a canine bladder defect model. Methods: We studied four healthy adult beagles (1-2 years old, 9.3-9.9 kg). Autologous Biosheets were prepared by embedding specially designed molds into subcutaneous pouches in the beagles. Eight weeks after implantation, the molds were extracted, and collagenous connective tissues surrounding the molds were harvested as autologous Biosheet. The urinary bladder wall was excised $(2 \mathrm{~cm} \times 2 \mathrm{~cm})$ and autologous Biosheets were sutured to the cut edge of the native bladder using a simple continuous suture pattern. The efficacy of implantation of the Biosheets was evaluated by physical examination, blood tests, abdominal ultrasound, urinalysis, and urography, at $0,1,3,7,14,28,56$, and 84 days after the implantation. The Biosheets were extracted at 28 days $(n=1)$ and 84 days $(n=3)$ after implantation. Results: No side-effects were observed during follow-up. No disruption of the sheet or any urinary leakage into the peritoneal cavity was observed. Histological analysis revealed a-SMA-positive muscle fibers at the margin of the Biosheets, indicating regeneration of the urinary bladder tissue. Conclusion: This is the first report evaluating the efficacy and safety of iBTAinduced autologous "Biosheets" as a bladder repair material in a canine model. In summary, autologous Biosheets could be useful biomaterials for urological reconstruction.

\section{Background}

Urinary bladder disorders, such as congenital abnormalities, cancer, inflammation, infection, trauma, innervation defects and iatrogenic injuries, often require reconstructive surgery $(1,2)$. Bladder augmentation with the small bowel, large bowel or stomach is commonly used in clinical applications $(3,4)$. However, surgical problems using gastrointestinal tissues can give rise to a wide variety of complications including mucus production, metabolic abnormalities, urinary tract infections, stone formation, intestinal obstruction and an increase in malignancies $(1,5,6)$. Furthermore, bladder reconstruction using autologous gastrointestinal tissues is invasive. It is therefore necessary to develop better suited implants for bladder reconstruction and regeneration.

Although artificial materials such as polytetrafluoroethylene (Gore-Tex), silicone or polyurethane can be produced reproducibly on a large scale with specified properties of strength, degradation rate, and microstructure, these materials may also lead to chronic inflammatory reactions and promote urinary tract infections and calcification $(7,8)$.

Tissue engineering techniques involve a combination of cells, engineering, and materials to overcome these complications in bladder reconstruction. Naturally derived biomaterials, including porcine small 
intestinal submucosa, bladder acellular matrix, amniotic membrane and others have been studied for their effectiveness as scaffolds for bladder augmentation (9-12). Hybrid biomaterials, using combinations of naturally derived scaffolds and synthetic polymers have also been explored to create novel biomaterials for regenerative medicine $(13,14)$.

Tissue engineering techniques incur cost for in-vitro cell management procedures or exceptionally clean laboratory facilities, both of which are expensive and labor-intensive over long periods. We advocate the use of in-body tissue architecture (iBTA); this technology has ushered in a new phase of tissue engineering techniques (15). It does not require complex in-vitro cell management procedures or exceptionally clean laboratory facilities. The grafts can be autologous. Moreover, grafts formed by iBTA can be made in any shape or form via suitably designed distributions of molds: tubular connective tissue (Biotube), (16) membranous tissue (Biosheets), (17) and three dimensional heart valve shaped tissue (Biovalve) (18). These have been fabricated and implanted in several animal models with satisfactory function. Optimal scaffolds for bladder reconstruction require biocompability in order to support cell migration, growth, and differentiation, and have suitable mechanical properties to sustain repeated stretching and relaxation of the bladder, and do not elicit a detrimental host inflammatory response before regeneration occurs. (19). Our previous studies found that iBTA-induced autologous collagenous connective membranes are capable of self-restoration and growth and do not suffer immunological rejection after implantation (15-18). Moreover, iBTA-induced autologous Biosheets had similar burst strength to native diaphragms which increased after transplantation, and similar elasticity (17).

We therefore believe that Biosheet is an appropriate scaffold for bladder reconstruction. Nevertheless, iBTA-induced grafts have not yet been used in urological tissue reconstruction. In this study, the efficacy and safety of autologous Biosheets was evaluated as bladder repair materials.

\section{Methods}

\section{Animals}

Four healthy adult beagles were selected for this study (1-2 years old, $9.3-9.9 \mathrm{~kg})$. These dogs were obtained from Oriental Yeast Co., Ltd and were housed individually in the stainless-steel cages in controlled environment with free access to water and food until 12 hours before anesthesia. Fig. 1 summarized the animal procedure schedule. The research protocols were approved by the ethics committee of Osaka Prefecture University animal experience (No. 29-81, 29-169).

\section{Preparation of Biosheets}

The mold for Biosheet preparation was assembled by inserting multiple stainless-steel slits into an acrylic tube (Fig. 2a). The Biosheets were prepared by embedding specially designed molds into subcutaneous pouches in the dogs $(n=4$, four molds per dog). Anesthesia was induced with propofol (8 $\mathrm{mg} / \mathrm{kg} \mathrm{IV})$, after premedication with atropine sulfate $(0.025 \mathrm{mg} / \mathrm{kg}$ Subcutaneously), butorphanol (0.2 $\mathrm{mg} / \mathrm{kg} \mathrm{IV})$, midazolam (0.2 mg/kg IV), and cefovecin sodium (8 mg/kg IV), and was maintained with $2 \%$ 
isoflurane in oxygen. Eight weeks after implantation, the molds were extracted (Fig. 2b), and collagenous connective tissue surrounding the molds was harvested as Biosheets (Fig. 2c).

\section{Surgical procedure}

After harvesting of the molds, the Biotubes formed on the acrylic tube were gathered by removing the molds and trimming the surrounding peripheral tissues. Rectangular-shaped Biosheets $(40 \times 50 \mathrm{~mm})$ were then obtained by trimming the Biotubes.

After trimming of the Biosheets, the dogs were repositioned. The urinary bladder was emptied before surgery using a urethral catheter. The urinary bladder was approached through a caudal midline abdominal incision. A full thickness piece of the urinary bladder wall was excised $(2 \mathrm{~cm} \times 2 \mathrm{~cm})$. Autologous Biosheets were sutured to the edge of the native bladder using a simple continuous suture pattern of 4-0 polydioxanone material (Fig. 3). Omentum was then placed over the Biosheets, before the abdominal incisions were routinely closed in three layers. The ureteral catheter was maintained for 4 hours postoperative and then remove.

\section{Evaluation of efficacy of Biosheets}

The efficacy of the implantation of the Biosheets was studied by physical examination, blood tests, abdominal ultrasound, urinalysis, and urography at $0,1,3,7,14,28,56$, and 84 days after implantation. Specific signs were monitored twice a day, including temperature, respiratory rate, pulse, appetite, activity, defecation and urination. The surgical wound was also observed and sterilized at the time of monitoring. Urine samples were collected by catheter at $0,7,14,28,56$, and 84 days after implantation. Urinalysis was performed to test for urine infection, or crystalluria. Abdominal ultrasound was performed to check for calculi, hematoma, hypertrophy and calcification in the reconstructed bladder. The dogs were given iohexol at doses of $600 \mathrm{mg}$ of iodine/ $1 \mathrm{~kg}$ of body weight by rapid IV injection; the urinary bladders of the dogs were empty prior to these injections. Ventrodorsal and lateral abdominal radiographs were made at $0,1,5,10,15$, and 20 minutes after injection of contrast medium, to check for leakage or diastolic dysfunction.

\section{Histological examination}

The Biosheets were extracted under anesthesia as mentioned above at 28 days $(n=1)$ and 84 days $(n=3)$ after implantation. After extracting of the Biosheets, the urinary bladders were sutured using a simple continuous suture pattern of 4-0 polydioxanone material. All of the dogs were rehabilitated and rehoused after this study. Extracted specimens were fixed with $10 \%$ formalin, embedded in paraffin, sliced into longitudinal sections, and stained with hematoxylin-eosin (HE) and masson-trichrome (MT). The specimens were also stained for a-smooth muscle actin ( $a-S M A)$ by immunohistochemical techniques with DAPI as nuclei counter staining; the protein was detected using monoclonal anti- bodies (Abcam, Cambridge, UK).

\section{Statistics}


All data are shown as median and range.

\section{Results}

\section{Summary of implantation of Biosheets}

Biosheets were successfully implanted in all four dogs for urinary bladder defects. The Biosheets were easy to suture onto the native urinary bladder, with no splitting at the anastomosis site. No perioperative complication was observed. Macroscopic viewing of the extracted Biosheets revealed that were covered with newly formed tissue at 84 days after the implantation (Fig. 4a and b).

\section{Evaluation of efficacy of Biosheets}

At the observation points of the experiment, all 4 dogs tolerated the procedure and survived. All had regular eating and activities. There were no obvious signs of complications such as severe gross hematuria or urine leakage. All 4 dogs exhibited patent urine excretion. Elevations in white blood cells and c-reactive protein levels were detected postoperatively (see Additional file 1). There was no hematuria, bacteria, or crystalluria in urinalysis throughout during the observation period (Table 1).

Urography and abdominal ultrasound did not show any urine leakage or bulging of Biosheets during the observation period (Fig. 5). There were no calculi, hematoma, or metaplasia in the urinary bladder according to abdominal ultrasound during any observation period (Fig. 5).

\section{Table 1. Summary of urinalysis in the observation periods.}

The urine samples collected with $0,7,14,28,56,84$ days after the implantation $(n=3)$. 


\begin{tabular}{|c|c|c|c|c|c|c|}
\hline $\begin{array}{l}\text { inspection } \\
\text { items }\end{array}$ & 0 & 7 & 14 & 28 & 56 & 84 \\
\hline \multirow[t]{3}{*}{ specific gravity } & 1.022 & 1.022 & 1.026 & 1.020 & 1.027 & 1.029 \\
\hline & (1.015- & (1.005- & (1.01- & $(1.01-$ & (1.021- & (1.005- \\
\hline & 1.025) & 1.04) & 1.037) & 1.029) & 1.03) & 1.053) \\
\hline \multirow[t]{2}{*}{$\mathrm{pH}$} & 6.7 & 7.2 & 6.8 & 8.1 & 6.8 & 7.7 \\
\hline & (6-7) & $(6.5-8.5)$ & $(5.5-8.5)$ & $(7-9)$ & $(6-7.5)$ & $(6-8.5)$ \\
\hline Bacteria & प & 口 & प & 口 & प & प \\
\hline Crystalluria & प & $\square$ & प & $\square$ & प & प \\
\hline
\end{tabular}

\section{Histological examination}

At 28 days after implantation, a multi-cell layer of the regenerated transitional epithelium completely covered the graft forming the mucosa of the substituted portion (Fig. 6). Neovascularization was observed in the loose connective tissue on the luminal (submucosa) (Fig. 6). Immunohistological analysis showed that smooth muscle cells had infiltrated at the marginetween the native bladder and the Biosheets at 84 days after implantation (Fig. 7), and infiltrated the substituted portion of the bladder. There was no calcification, necrosis or malignance at 28 days and 84 days after implantation.

\section{Discussion}

In this study we have demonstrated the implantation of autologous Biosheets, produced by iBTA technology in the urinary bladder, as urological reconstruction materials. Our results indicate that Biosheets can be used for repairing bladder defects without complication, and one in process of regenerating muscular tissue at 84 days after implantation.

In terms of bladder regeneration, present surgical procedures utilizing bowel segments give rise to numerous complications, including mucus production, chronic bacteriuria, ruptures, leakages and fibrosis; 
the main complication is stone formation due to exposure to urine for even a short period of time (19) The calculus was composed of ammonium magnesium phosphate, and may be caused by urinary infection (20). No bacteria were found in urinalysis during the observation period in any of the dogs. There was no stone formation in bladder reconstruction using Biosheets. Furthermore, no dogs had no urine leakage or bulging of the Biosheets. Our result suggest that Biosheets can be a useful scaffold for urological reconstruction without subsequent complications.

The urothelium cells lining the inner surface of the detrusor smooth muscle layer of the bladder has crucial roles beyond providing an essential barrier function to protect the detrusor from urine (21). A previous report found that urinary bladder smooth muscle engineered from adipose stem cells, and a three-dimensional synthetic composite after 4 weeks in vivo, had an organized urothelium covering the entire lumen of the graft (22). Histological examination revealed that the entire Biosheet was coavered with urothelium cells at 28 days after implantation. Biosheets are therefore useful as an appropriate scaffold of migration and proliferation for urothelium cells.

In this report, aSMA positive cells were observed mainly at the margins between native urinary bladder muscle tissue and Biosheets. The result shows that Biosheets comprises a suitable scaffold for migration of native smooth muscle cells and extending muscle tissues. We have previously found that Biosheets produced by iBTA technology can recruit tissue stem cells from native tissue, and that these stem cells then mature and undergo proliferation (17). Biosheets were reconstructed in a manner analogous to native tissue, such as cornea, trachea, diaphragm, and esophagus (17, 23-25). Following long observation, those cells have the capability to mature and construct muscular tissue similar to native bladder smooth muscle tissue.

We conducted this experiment on dogs because this technique of transplantation be used clinically in canines. This relatively simple and less expensive method has the advantage of using available autologous Biosheets for urological reconstruction without complication. Several limitations of this study are apparent. First, this study was a short-term follow-up. Second, it was not a comparative investigation. Owing to the limited number of animals used, this study is only suggestive, but nevertheless provides valuable insights into urological reconstruction. Additional studies, including assessments of long-term follow-up, will be necessary in order to fully evaluate the potential of this bioscaffold-based regenerative medicine method for urological reconstruction.

\section{Conclusion}

This is the first report evaluating the efficacy and safety of iBTA-induced autologous "Biosheets" as a bladder repair material in a canine model. In conclusion, our results showed that autologous Biosheets can be valuable as repair materials for bladder reconstruction.

\section{Abbreviations}


iBTA: in body tissue architecture; HE: hematoxylin-eosin; MT: masson-trichrome; a-SMA: a-smooth muscle actin

\section{Declarations}

\section{Acknowledgements}

There were no particular people or institutions to be acknowledged during this study.

\section{Authors' contributions}

YI: study design, surgical operation, data collection, manuscript writing, data analysis, management, NK: surgical operation, study design, data collection, data analysis, RI pathological evaluation, data collection, data analysis, KN: surgical operation, data collection, data analysis, YI: surgical operation, data collection, data analysis, MF: surgical operation, data collection, data analysis, MO: surgical operation, data collection, data analysis, MN: data collection, data analysis, KM: surgical operation, data collection, data analysis, $\mathrm{HN}$ : data collection, supervision, management, YN: study design, data collection, data analysis, management, supervision, HA: study design, surgical operation, data collection, manuscript writing, data analysis, management.

All authors read and approved the final manuscript.

\section{Funding}

No financial support was received for this work.

\section{Availability of data and consent to participate}

The datasets used and/or analyzed during the current study available from the corresponding author on reasonable request.

\section{Ethics approval and consent to participate}

The research protocols were approved by the ethics committee of Osaka Prefecture University animal experience (No. 29-81, 29-169)

\section{Consent for publication}

Not applicable.

\section{Competing interests}

The authors declare that they have no competing interests.

\section{Author details}


${ }^{1}$ Laboratory of Veterinary Surgery, Graduate School of Life and Environmental Sciences, Osaka Prefecture University, Izumisano, Osaka, Japan

${ }^{2}$ Institute of Frontier Science and Technology, Okayama University of Science, Okayama, Okayama, Japan

${ }^{3}$ Biotube Co., Ltd., Chuo, Tokyo, Japan

\section{References}

1. Biers SM, Venn SN, Greenwell TJ. The past, present and future of augmentation cystoplasty. BJU Int. 2012;109(9):1280-93.

2. Kim KH. The evolution of urogenital tissue engineering. Int Neurourol. J. 2011;15(3):107-8.

3. Pokrywczynska M, Adamowicz J, Sharma AK, Drewa T. Human urinary bladder regeneration through tissue engineering - an analysis of 131 clinical cases. Exp Biol Med (Maywood). 2014;239(3):264-71

4. Langer S, Radtke C, Györi E, Springer A, Metzelder ML. Bladder augmentation in children: current problems and experimental strategies for reconstruction. Wien Med Wochenschr. 2019;169(3-4):6170.

5. Mingin GC, Stock JA, Hanna MK. Gastrocystoplasty: long-term complications in 22 patients. J Urol. 1999 Sep;162(3 Pt 2):1122-5.

6. Kurzrock EA. Pediatric enterocystoplasty: Iong-term complications and controversies. World J Urol. 2009;27(1):69-73.

7. Senel FM, Akman RY, Arikan AY, Gürdal M, Can C The use of polytetrafluoroethylene (Gore-Tex) grafts in reconstruction of the urinary bladder. Int Urol Nephrol. 1999;31(3):313-9.

8. Noe HN, Williams RS, Causey J, Smith DP. Long-term effects ofpolytetrafluoroethylene injectedinto the rat bladder submucosa. urology. 1994;43(6):852-5.

9. Petrovic V, Stankovic J, Stefanovic V. Tissue engineering of the urinary bladder: current concepts and future perspectives. ScientificWorldJournal. 2011;11:1479-88.

10. Sharma AK, Cheng EY. Growth factor and small molecule influence on urological tissue regeneration utilizing cell seeded scaffolds. Adv Drug Deliv Rev. 2015;82-83:86-92.

11. Adamowicz J, Pokrywczynska M, Tworkiewicz J, Kowalczyk T, van Breda SV, Tyloch D, et al. New Amniotic Membrane Based Biocomposite for Future Application in Reconstructive Urology. PLoS One. 2016;11(1):e0146012.

12. Leonhauser D, Stollenwerk K, Seifarth V, Zraik IM, Vogt M, Srinivasan PK, et al. Two differentially structured collagen scaffolds for potential urinary bladder augmentation: proof of concept study in a Gottingen minipig model. J Transl Med. 2017;15(1):3.

13. Horst M, Madduri S, Milleret V, Sulser T, Gobet R, Eberli D.A bilayered hybrid microfibrous PLGAacellular matrix scaffold for hollow organ tissue engineering. Biomaterials. 2013;34(5):1537-45. 
14. Horst M, Milleret V, Nötzli S, Madduri S, Sulser T, Gobet R, Eberli D. Increased porosity of electrospun hybrid scaffolds improved bladder tissue regeneration. J Biomed Mater Res A. 2014;102(7):2116-24.

15. Hayashida K, Kanda K, Yaku H, Ando J, Nakayama Y. Development of an in vivo tissue-engineered, autologous heart valve (the biovalve): preparation of a prototype model. J Thorac Cardiovasc Surg. 2007;134(1):152-9.

16. Watanabe T, Kanda K, Yamanami M, Ishibashi-Ueda H, Yaku H, Nakayama Y. Long-term animal implantation study of biotube-autologous small-caliber vascular graft fabricated by in-body tissue architecture. J Biomed Mater Res B Appl Biomater. 2011;98(1):120-6.

17. Suzuki K, Komura M, Terawaki K, Kodaka T, Gohara T, Komura $H$, et al. Engineering and repair of diaphragm using biosheet (a collagenous connective tissue membrane) in rabbits. J Pediatr Surg. 2018;53(2):330-4.

18. Funayama M, Matsui Y, Tajikawa T, Sasagawa T, Saito Y, Sagishima S, et al. Successful implantation of autologous valved conduits with self-expanding stent (stent-biovalve) within the pulmonary artery in beagle dogs. J Vet Cardiol. 2015;17(1):54-61.

19. Lin HK, Madihally SV, Palmer B, Frimberger D, Fung KM, Kropp BP. Biomatrices for bladder reconstruction. Adv Drug Deliv Rev. 2015;82-83:47-63.

20. Prywer J, Olszynski M. Bacterially induced formation of infectious urinary stones: recent developments and future challenges. Curr Med Chem. 2016; 24:292-311.

21. Fry $\mathrm{CH}$, Vahabi B. The Role of the Mucosa in Normal and Abnormal Bladder Function. Basic Clin Pharmacol Toxicol. 2016;119 Suppl 3:57-62.

22. Jack GS, Zhang R, Lee M, Xu Y, Wu BM, Rodríguez LV.Urinary Bladder Smooth Muscle Engineered from Adipose Stem Cells and a Three Dimensional Synthetic Composite. Biomaterials 2009;30(19):3259-70.

23. Okuyama H, Umeda S, Takama Y, Terasawa T, Nakayama Y. Patch esophagoplasty using an in-bodytissue-engineered collagenous connective tissue membrane. J Pediatr Surg. 2018;53(2):223-6.

24. Takiyama N, Mizuno T, Iwai R, Uechi M, Nakayama Y. In-body tissue-engineered collagenous connective tissue membranes (BIOSHEETs) for potential corneal stromal substitution. J Tissue Eng Regen Med. 2016;10(10):E518-E26.

25. Satake R, Komura M, Komura H, Kodaka T, Terawaki K, Ikebukuro K, et al. Patch tracheoplasty in body tissue engineering using collagenous connective tissue membranes (biosheets). J Pediatr Surg. 2016;51(2):244-8.

\section{Additional File}

Additional file 1 Summary of blood tests in observation periods.

The blood samples collected at $0,1,7,14,28,56,84$ days after implantation $(n=3)$.

\section{Figures}




\section{Adult beagle dogs 우 $(n=4)$}

\section{(DAY)-56

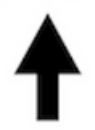

Molds embed

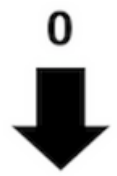

Autologous Urinary bladder Biosheets reconstruction

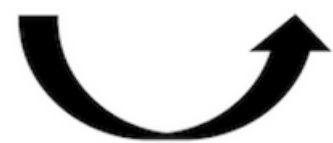

$\begin{array}{lll}1 & 3 & 7\end{array}$

14

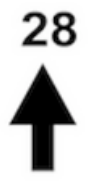

Histological examination $(n=1)$

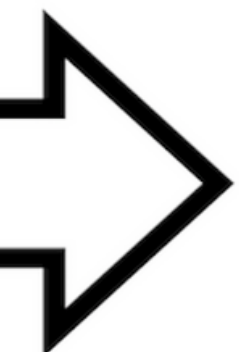

56

84

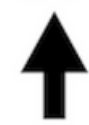

Histological examination $(n=3)$

\section{Figure 1}

Animal procedure schedule.

A

\section{Figure 2}

Photograph of the mold and Biosheet. (a) Specially designed mold for the Biosheet. (b) Appearance of the mold extracted at 8 weeks after implantation. (c) Gross appearance of collagenous connective tissue membrane (Biosheet).

层

\section{Figure 3}

Macroscopic view of implantation of Biosheets in urinary bladder.

2

Figure 4

Macroscopic view of extracting of Biosheets in urinary bladder. (a)Photograph of urinary bladder at 84 days after implantation. (b) Mucous membrane of Biosheets at 84 days after implantation. 

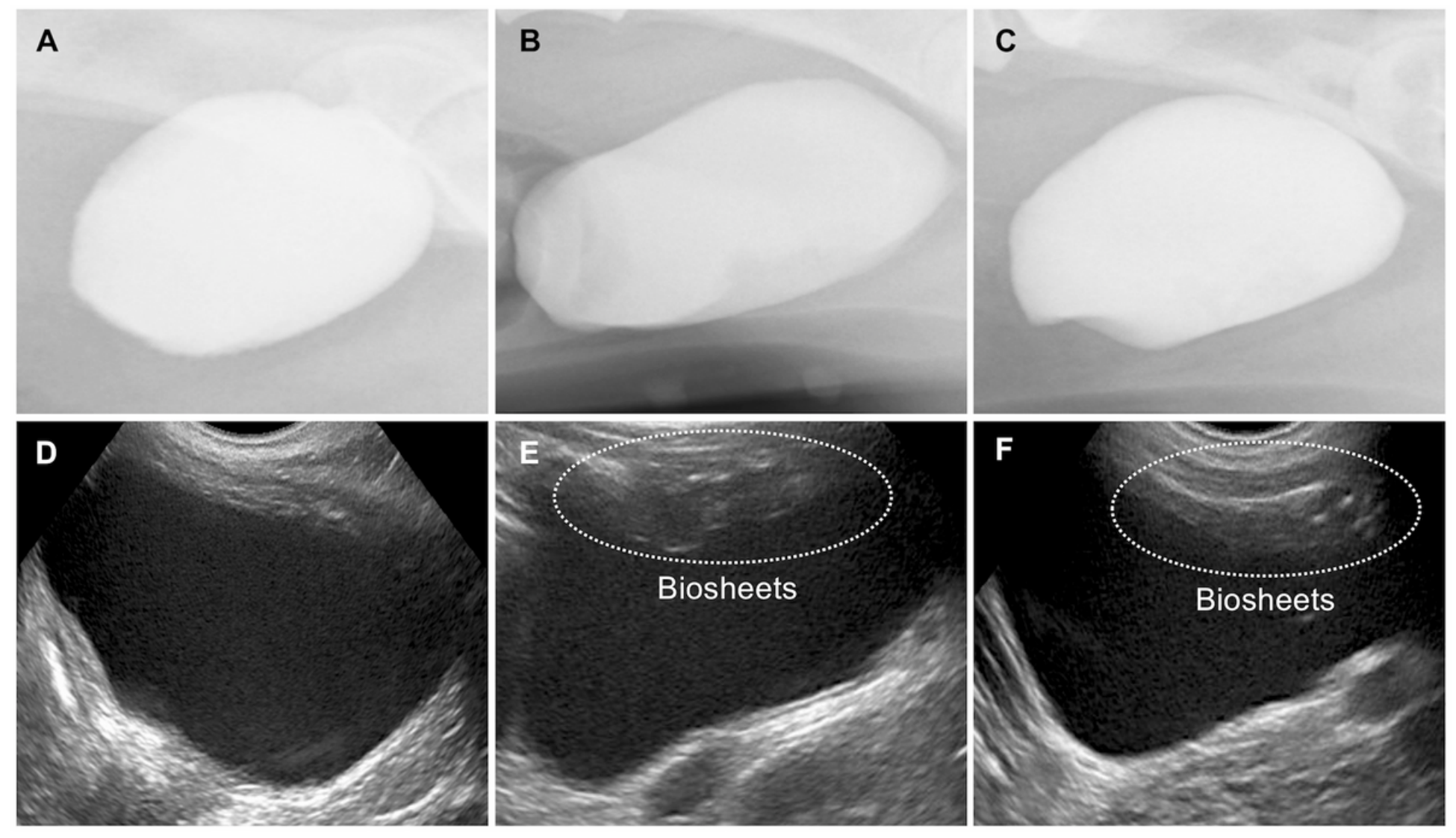

\section{Figure 5}

Representative images of urography and abdominal ultrasound after the implantation of Biosheets. (a, d) Images of the urinary bladder at 0 days after the implantation. (b, e) Images of the urinary bladder at 28 days after the implantation. (c, f) Images of the urinary bladder at 84 days after the implantation.

\section{Figure 6}

Histology of Biosheets at 28 days after implantation. (a) Hematoxylin-eosin staining. Entire view of Biosheets. (b) Masson's Trichrome staining. Collagen is colored blue, nuclei colored black. Stratified urothelium covered the entire graft surface of the Biosheets (black arrows). Neovascularization was observed in the loose connective tissue on the luminal (white arrows).

\section{Figure 7}

Histology of Biosheets at 84 days after implantation. (a) Entire view of Biosheets. (b, c) Immunohistochemical staining for a-smooth muscle actin (a-SMA). Red: a-SMA, Blue: DAPI staining for cell nuclei. There were cells which were stained positive for aSMA recruited from native muscle tissues to Biosheets. 


\section{Supplementary Files}

This is a list of supplementary files associated with this preprint. Click to download.

- Additionalfile1.pdf

- NC3RsARRIVEGuidelinesChecklist2014forBMCurology.pdf 